\section{Primary neurofibroma of the common bile duct: a case report}

Dominique Béchade, ${ }^{1}$ Thierry Boulanger, ${ }^{2}$
Laurent Palazzo, ${ }^{3}$ Jean-Pierre Algayres'

${ }^{1}$ Department of Medicine, Cancer

Research Institute, Bordeaux (Institut

Bergonié); ' Department of Surgery, Val de Grâce, Paris; ${ }^{3} 30$ Rue d'Astorg, Paris, France

\section{Abstract}

Obstructive jaundice secondary to common bile duct stricture is attributed most commonly to pancreatic cancer and cholangiocarcinoma. Benign tumors of the extrahepatic biliary duct such as papilloma or adenoma are rare, accounting for $6 \%$ of biliary tumors. ${ }^{1,2}$ Among them, neurofibromas of the extrahepatic biliary tract are extremely rare and clinically nonspecific. Their preoperative diagnosis is most difficult, especially when there is no history of cholecystectomy or biliary tract trauma. ${ }^{3,4} \mathrm{We}$ report a case of such a lesion in a young female patient.

\section{Case Report}

A 28-year-old female patient was referred for upper abdominal pain and fever $\left(38.5^{\circ} \mathrm{C}\right)$ that had been evolving over eight days. She had no family history of a hereditary disorder, nor personal previous history of cholecystectomy or trauma of the bile duct. The physical examination was normal, except for tenderness in the right hypochondrium. There was no evidence of jaundice. The laboratory studies revealed: serum alanine aminotransferase (ALAT), 54 IU/L (normal <52 IU/L); serum aspartate aminotransferase (ASAT), 48 IU/L (normal $<36$ IU/L); total bilirubin, normal; serum alkaline phosphatase, 157 IU/L (normal <126 IU/L); serum $\gamma$-glutamyl transpeptidase, 40 IU/L (normal <78 IU/L); C-reactive protein (CRP), 140 $\mathrm{mg} / \mathrm{L}$ (normal $<8 \mathrm{mg} / \mathrm{L}$ ); leukocyte count, $14.7 \times 10^{9} / \mathrm{L}$; neutrophil count, $9.7 \times 10 / \mathrm{L}$.

Abdominal ultrasonography showed mild hepatomegaly and slight dilatation of the intrahepatic biliary tracts, which seemed to be secondary to adenopathies of the hepatic hilum measuring $15.8 \mathrm{~mm}$ and $12.9 \mathrm{~mm}$. Abdominal computed axial tomography (CT) (Figure 1) showed a well-demarcated, homogeneous and hypodense mass (20-35 UH) on unenhanced scans, with no enhancement after injection of the contrast medium. The mass surrounded the entire common bile duct and invaded the hepatic hilum, without any dilatation of the intrahepatic biliary tracts and without adenopathy. Abdominal magnetic resonance imaging (MRI) (Figure 2) showed a tissue mass with very clear borders, infiltrating the hepatic hilum. This mass was pushing the common bile duct back, sheathing the right and particularly the left portal pedicles as well as the bile duct, with no dilatation of the intrahepatic bile ducts and no mass effect on the blood vessels. This lesion had low signal intensity on T1-weighted images with moderate and heterogeneous signal intensity on T2-weighted images: it seemed to contain more hyperintense lesions on T2-weighted images. The contrast-enhanced T1-weighted images showed no enhancement. The endoscopic ultrasound (Figure 3) revealed multiple, oval, coalescent masses at the hepatic pedicle, particularly in front of and behind the portal vein; the largest of these masses had an axis of $3 \mathrm{~cm}$. No fine needle aspiration was carried out on these lesions.

Evolution of pain and fever was spontaneously favorable without any treatment, especially antibiotics. There was no explanation of the fever after physical and laboratory examinations. The endoscopic ultrasound ruled out any stones in the gall bladder and common bile duct, which could explain such a high fever. Endoscopic retrograde cholangiography was not performed.

Assuming that a low-grade lymphoma, sar-
Correspondence: Dominique Béchade, Institut Bergonié, 229 Cours de l'Argonne, 33076 Bordeaux Cedex, France.

E-mail: dominique.bechade94@orange.fr

Key words: neurofibroma, common bile duct, endoscopic ultrasound.

Received for publication: 6 September 2009.

Revision received: 20 0ctober 2009.

Accepted for publication: 22 October 2009.

This work is licensed under a Creative Commons Attribution 3.0 License (by-nc 3.0).

@C Copyright D. Béchade et al., 2009

Licensee PAGEPress, Italy

Gastroenterology Insights 2009; 1:e6 doi:10.4081/gi.2009.e6

coidosis, an inflammatory pseudotumor of the ganglion, or ganglionic tuberculosis was involved, we performed a surgical biopsy under laparoscopy and finally diagnosed a primary neurofibroma of the common bile duct (Figures 4 and 5). The tumors were removed surgically. Three years later the patient remains asymptomatic, with normal clinical examination and biological liver test results. Echography shows the persistence of the conglomerate, non-compressive hepatic pedicle lesions, measuring between $5 \mathrm{~mm}$ and $17 \mathrm{~mm}$.

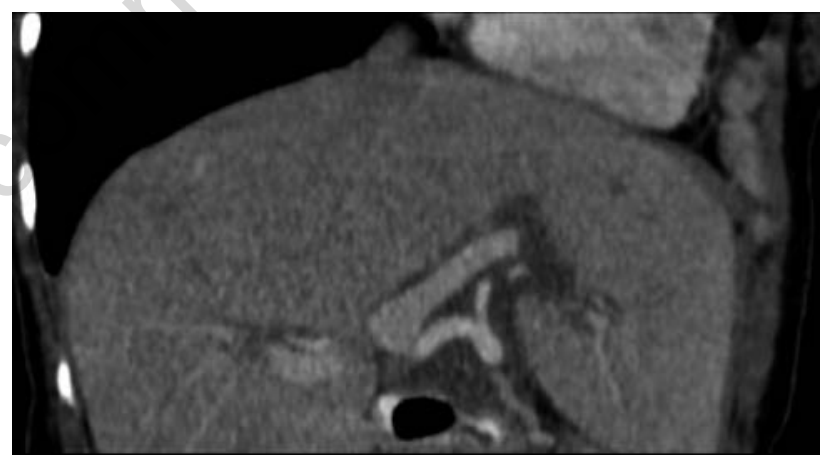

Figure 1. Pre-contrast abdominal computed tomography scan showing a well-demarcated homogenous mass. The mass is not enhanced on post-contrast CT.
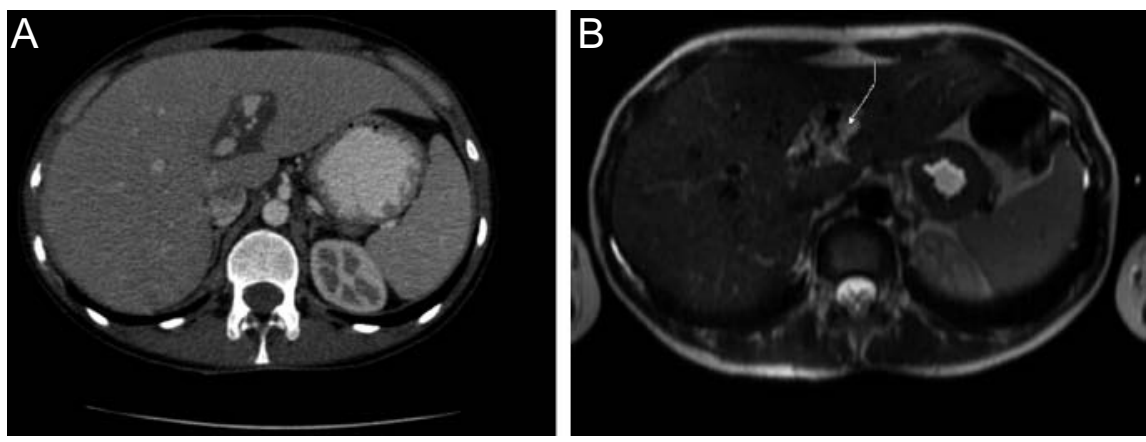

Figure 2. (A) and (B) T2-weighted magnetic resonance images showing a heterogeneous mass containing more hyperintense regions $(\mathrm{B}$, arrow). 


\section{Discussion}

Neurofibroma, sometimes known as neurilemmoma or schwannoma, are rare benign tumors derived from the Schwann cells of nerve sheaths. ${ }^{3,5}$ They usually arise in the soft tissues of the head, neck, spinal cord, mediastinum, and extremities because they occur along the course of a peripheral, cranial, or sympathetic nerve. ${ }^{5}$ In the digestive system, they may arise in the gastrointestinal tract and the liver. ${ }^{5}$ As they represent only $6 \%$ of primary retroperitoneal tumors, rare cases in the common bile duct have been reported..$^{5}$ ${ }^{7}$ They can develop occasionally in the setting of type 2 neurofibromatosis. ${ }^{7,8}$ In our case report no familial or personal history of neurofibromatosis was found.

Extra-hepatic bile duct neurofibromas originate in the sympathetic and parasympathetic fibers of the wall of the common bile duct. ${ }^{5}$ Two kinds have been described:

1. Traumatic neurofibromas are characterized by the proliferation of nerve fibers in the connective tissue surrounding a nerve, where the epineurium has been ruptured during operative trauma to the common bile duct. The extra-hepatic bile ducts are exposed particularly to this kind of post-traumatic lesion, owing to their significant neurovegetative innervation. The sympathetic and parasympathetic fibers from the coeliac plexus converge toward the cystic duct. They develop at the cystic duct remnant or in the wall of the hepatic duct. In the common bile duct, they usually are described as post-cholecystectomy amputation neurofibromas ${ }^{3,9}$ or described after orthotopic liver transplantation..$^{10} \mathrm{~A}$ case report of a neurofibroma of the common bile duct, at a follow-up 15 years after surgical treatment of a type Ic Todani choledochal cyst, with an associated anomaly of the biliopancreatic junction, by choledocojejunostomy with Y-en-Roux anastomosis, forms a singular pathophysiological model. ${ }^{11}$ The quasiexclusive location of the common bile duct and the cyst at the head of the pancreas having made bile derivation necessary, the neurofibroma was formed by chronic reflux of pancreatic fluid into the residual cyst.

2. Primary neurofibromas, as in this case report, in which axonal proliferation remains confined to the inside of the epineurium. These are much more rare ${ }^{12,13}$ and may be the result of chronic inflammatory lesions, infections, granulomatous reactions to foreign bodies, or ischemic lesions. ${ }^{12}$ They can be associated with biliary stones, ${ }^{5}$ as it seems that bile stasis owing to mechanical obstruction of the bile duct by the neurinoma can encourage lithogenesis.

The sex ratio is two women to one man, and these tumors occur at an average age. ${ }^{4}$

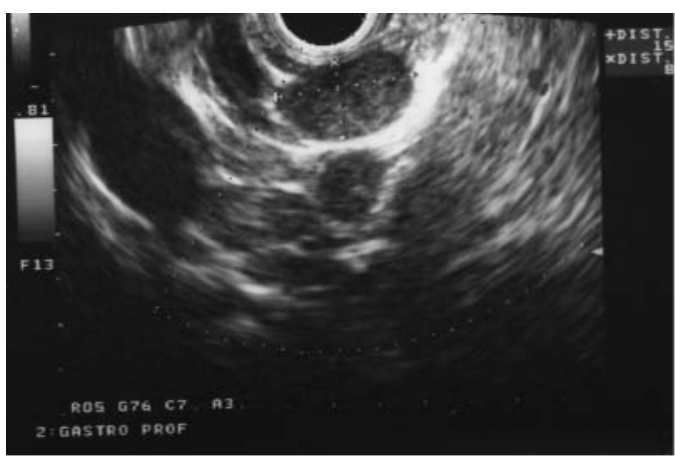

Figure 3. Endoscopic ultrasonography showing oval adenopathies.

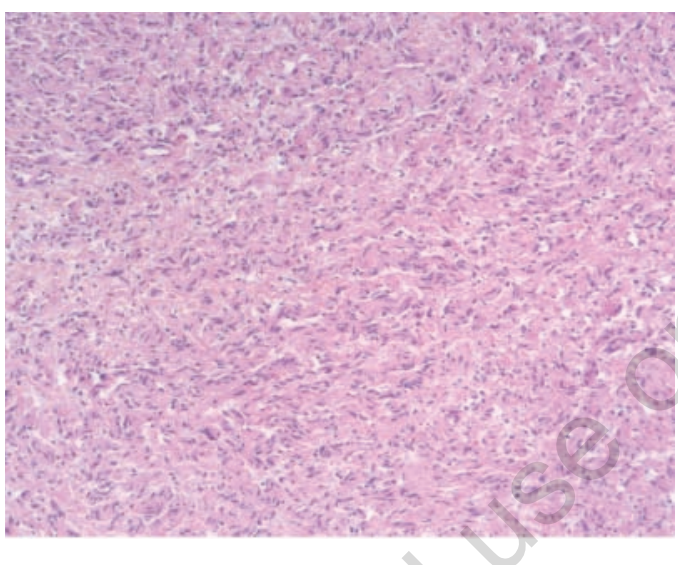

Figure 4. Microscopic examination proved the tumor of the common bile duct to be a benign neurofibroma, which was composed chiefly of long, spindle-shaped fibrous cells (hematoxylin and eosin stain, 100X magnification).

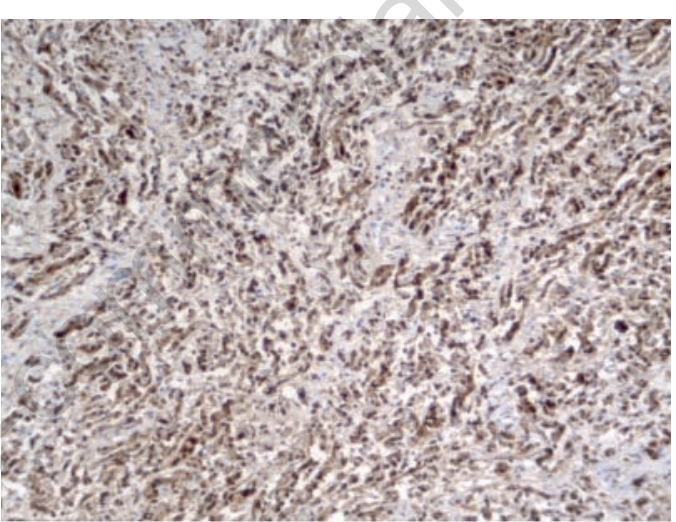

Figure 5. Immunohistochemically, the cytoplasm and nuclei of the spindle-shaped tumor cells appear reactive for $S-100$ protein (brown) (100X magnification).

Little is known about these neurogenic tumors in the biliary system, because most of them seem to be asymptomatic. ${ }^{4}$ When symptomatic, symptoms are caused by the compression of adjacent structures; ${ }^{5}$ thus, jaundice is the clinical sign that is described most often in the majority of reported case histories. ${ }^{1,2,4,5,12,13}$ The non-jaundice symptomatic forms, as in this case report, are exceptional. ${ }^{14}$ Febrile pain in the right hypochondrium with spontaneously favorable evolution reveals the nature of this type of lesion, which probably is intermittently compressive. This physiopathological data may explain such a high fever in our patient.

Preoperative diagnosis of primary neurofibroma of the bile duct is difficult; scanning reveals a homogeneous mass with heteroge- neous enhancement and occasional cyst cavities, necrotic areas, or calcifications. ${ }^{15}$ Magnetic resonance imaging findings are masses with low signal intensity on the T1weighted images and inhomogeneous high signal intensity on the T2-weighted images, probably owing to a partial fluid content, ${ }^{16}$ or no enhancement as in this case. Endoscopic ultrasound data are scarce; the image described corresponds to a solid tumor of the non-vascular peri-bile duct, containing multiple anechogenic areas that are cystic in appearance. ${ }^{4,5}$ These data are very different to the misleading pseudo-ganglionic image observed in this case. Surgery was necessary because of the impossibility of ruling out a cholangiocarcinoma on the basis of the preoperative radiological examinations and the 
need to prevent the installation of biliary cirrhosis secondary to this benign lesion. ${ }^{2}$

Macroscopically there were one or several tumors, which were ovoid and encapsulated with clear borders, as in this case report. An intraoperative pathology consultation is essential, in order to avoid a cephalic duodenopancreatectomy or a hepatic hilar resection for cancer. ${ }^{2}$ This was the approach chosen in this case, which led to enucleation and decompression of the common bile duct. Pathologically the lesion was composed chiefly of long spindle-shaped cells, whose neurogenic character was confirmed by positive immunohistochemistry for neurofilament protein. Furthermore, there was no nuclear heteromorphism, which suggests a benign nature. Neurofibromas do not express CD34, CD117, and muscle markers, and thus are differentiated from stromal tumors. The neurofibroma diagnosis established during the operation led to an exploration of the other intra-abdominal organs during the operation, based on the hypothesis of type 2 neurofibromatosis. ${ }^{17}$

Neurofibroma, a benign neurogenic lesion of the bile duct, mimics more common causes of bilious obstruction and presents preoperative diagnosis difficulties; it is important to consider it in cases where there is a history of bile duct intervention, particularly cholecystectomy. Treatment of primary neurofibroma should, as in this case report, be guided by an extemporaneous anatomopathological examination, allowing a simple surgical act to be carried out.

\section{References}

1. Peyre CG, Wakim M, Mateo R, et al. Unusual cases of jaundice secondary to non-neoplastic bile duct obstruction. Am Surg 2004;70:620-4.

2. Li FY, Cheng JQ, He S, et al. Primary neurofibroma of the common bile duct as an unusual cause of obstructive jaundice: a case report. Dig Dis Sci 2005;50:1166-8.

3. Capovilla M, Lazure T, Lorand I, et al. Post-cholecystectomy amputation neuroma of the common bile duct with obstructive jaundice. Gastroenterol Clin Biol 2005;29: 80-2.

4. Fenoglio L, Severini S, Cena P, et al. Common bile duct schwannoma: A case report and review of literature. World $\mathbf{J}$ Gastroenterol 2007;13:1275-8.

5. Honjo Y, Kobayashi Y, Nakamura T, et al. Extrahepatic biliary schwannoma. Dig Dis Sci 2003;48:2221-6.

6. Lane RH, Stephens DH, Reiman HM. Primary retroperitoneal neoplasms: CT findings in 90 cases with clinical and pathologic correlation. Am J Roentgenol 1989;152:83-9.

7. Wiermann H, Wienbeck M. Benign tumours as the cause of extrahepatic occlusion syndrome. Report on a neuroma of the bile ducts. Z Gastroenterol 1975;13: 685-9.

8. MacCollin M, Chiocca EA, Evans DG, et al. Diagnostic criteria for schwannomatosis. Neurology 2005;64:1838-45.
9. Hotta T, Kobayashi Y, Taniguchi K, et al. A traumatic neuroma of the bile duct: a case report. Hepato-Gastroenterology 2004;51: 39-42.

10. Colina F, Garcia-Prats MD, Moreno E, et al. Amputation neuroma of the hepatic hilum after orthotopic liver transplantation. Histopathology 1994;25:151-7.

11. Otani T, Shioiri T, Mishima H, et al. Bile duct schwannoma developed in the remnant choledochal cyst - a case associated with total agenesis of the dorsal pancreas. Dig Liver Dis 2005;37:705-8.

12. Sugahara K, Yamamoto $M$, lizuka $\mathrm{H}$, et al. Spontaneous neuroma of the bile duct: A case report. Am J Gastroenterol 1985;80: 807-9.

13. Peison B, Benisch B. Traumatic neuroma of the cystic duct in the absence of previous surgery. Hum Pathol 1985;11:1168-9.

14. Jung JH, Joo KR, Chae MJ, et al. Extrahepatic biliary schwannomas: a case report. J Korean Med Sci 2007;22:549-52.

15. Levy AD, Quiles AM, Miettinen M, et al. Gastrointestinal schwannomas: CT features with clinicopathological correlation. Am J Roentgenol 2005;184:797-802.

16. Rha SE, Byun JY, Jung SE, et al. Neurogenic tumors in the abdomen: tumor types and imaging characteristics. Radiographics 2003;23:29-43.

17. Bajor J, Garamszegi M, Grexa E, et al. Bile duct obstruction caused by neurofibroma in a patient with Recklinghausen's disease. Orv Hetil 2002;143: 1947-50. 